\title{
Impact of Rheumatoid Arthritis on Alopecia: A Nationwide Population-Based Cohort Study in Taiwan
}

\author{
Yi-Jung Chang ${ }^{1,2}$, Yung-Heng Lee ${ }^{3,4,5,6}$, Pui-Ying Leong ${ }^{7,8,9}$, Yu-Hsun Wang ${ }^{10}$ and \\ James Cheng-Chung Wei ${ }^{7,8,9,11 *}$
}

1 Department of Pediatrics, Chang Gung Memorial's Hospital, Taoyuan, Taiwan, ${ }^{2}$ Chang Gung University College of Medicine, Taoyuan, Taiwan, ${ }^{3}$ Department of Health Services Administration, China Medical University, Taichung, Taiwan, ${ }^{4}$ Department of Public Health, China Medical University, Taichung, Taiwan, ${ }^{5}$ Department of Orthopedics, Cishan Hospital, Ministry of Health and Welfare, Kaohsiung, Taiwan, ${ }^{6}$ Department of Center for General Education, National United University, Miaoli, Taiwan, ${ }^{7}$ Department of Rheumatology, BenQ Medical Center, The Affiliated BenQ Hospital of Nanjing Medical University, Taichung, Taiwan, ${ }^{8}$ Department of Allergy, Immunology and Rheumatology, Chung Shan Medical University Hospital, Taichung, Taiwan, ${ }^{9}$ Institute of Medicine, Chung Shan Medical University, Taichung, Taiwan, ${ }^{10}$ Department of Medical Research, Chung Shan Medical University Hospital, Taichung, Taiwan, ${ }^{11}$ Graduate Institute of Integrated Medicine, China Medical University, Taichung, Taiwan

\section{OPEN ACCESS}

Edited by:

Kayo Masuko,

Sanno Medical Center, Japan

Reviewed by:

Gianluca Bagnato,

University of Messina, Italy

Mihir D. Wechalekar,

Flinders Medical Centre, Australia

Vasco C. Romão,

Universidade de Lisboa, Portugal

*Correspondence:

James Cheng-Chung Wei jccwei@gmail.com

Specialty section:

This article was submitted to

Rheumatology,

a section of the journal

Frontiers in Medicine

Received: 10 January 2020

Accepted: 06 April 2020

Published: 28 April 2020

Citation:

Chang Y-J, Lee Y-H, Leong P-Y, Wang Y-H and Wei JC-C (2020) Impact of Rheumatoid Arthritis on Alopecia: A Nationwide Population-Based Cohort Study in

Taiwan. Front. Med. 7:150.

doi: 10.3389/fmed.2020.00150
Objectives: Studies on the relationship between rheumatoid arthritis (RA) and alopecia areata (AA) are limited. This study investigated the effect of RA on alopecia areata risk in a nationwide cohort study.

Methods: We analyzed 2000-2012 data from the Longitudinal Health Insurance Database in Taiwan. The follow-up period was extended up to the end of 2013. We defined RA as a diagnosis using International Classification of Diseases, Ninth Revision, Clinical Modification (ICD-9-CM) code 714.0 during at least three outpatient visits or one admission and the use of disease-modifying antirheumatic drugs (DMARDs) for $>30$ days. The enrollees with AA were identified using the ICD-9-CM code 704.01. We enrolled a comparison cohort comprising participants randomly matched by age and sex, with the same index date as that of the study cohort. Furthermore, we investigated alopecia risk by using Cox proportional-hazards regression models after propensity score matching for sex, age, comorbidities, and medication use.

Results: In total, 2,905 patients with RA (74\% women, mean age: 51.9 years) and 2,905 controls were followed for 22,276 and 25,732 person-years, respectively. Alopecia risk was 2.64 -fold (95\% confidence interval $=1.47-4.76$ ) higher in patients with RA than in patients without RA after age, sex, comorbidities, and medication use were adjusted for. In addition, patients with thyroid disease presented considerable alopecia risk. Patients with RA in the younger age group (20-40 years) had the highest alopecia risk.

Conclusions: Alopecia risk is significantly higher in patients with RA than in those without RA, particularly in the younger age group (20-40 years). RA assessment should be considered when examining patients with alopecia, especially young adults.

Keywords: alopecia, rheumatoid arthritis, cohort study, corticosteroids, disease-modifying antirheumatic drugs 


\section{INTRODUCTION}

Alopecia occurs worldwide, with a lifetime incidence of $\sim 2 \%$ in the general population (1). It is usually characterized by unpredictable, recurring, chronic, and nonscarring loss of hair on the scalp or other areas (2). Alopecia is not a cosmetic problem specifically because it is associated with several comorbidities that may affect the patients' overall health and cause considerable morbidity (3-5). According to epidemiologic studies, autoimmune disorders, including rheumatoid arthritis (RA), play a role in the comorbid condition associated with alopecia (6).

RA affects $0.5-1 \%$ of the general population (7). It is a progressive disease involving a chronic and abnormal inflammatory reaction of the immune system against body tissues (systemic), primarily attacking joints and possibly hair follicles, leading to hair loss (8). Alopecia may present early in patients with RA, even before the more classical form of RA is identified. Timely diagnosis and appropriate treatment by a multidisciplinary team of clinicians are crucial for preventing further manifestations and yielding beneficial longterm outcomes in patients.

However, epidemiological studies on the relationship between RA and alopecia are limited, with many such studies with the limitation of a small sample size. Moreover, no clear conclusions can be drawn from these studies. To fill this knowledge gap, we conducted a nationwide population-based cohort study to elucidate alopecia risk in patients with RA.

\section{METHODS}

\section{Data Source}

The present nationwide population-based study was based on data retrieved from the National Health Insurance (NHI) Research Database (NHIRD). The NHI program, established in 1995, covers $>98 \%$ of the Taiwanese population. NHIRD contains anonymized data linked from the NHI for epidemiological study, including patient demographic details; health care service data; medication dispensation data from hospitals, general practices, and community pharmacies; diagnostic codes of International Classification of Diseases, Ninth Revision, Clinical Modification (ICD-9-CM), and drug codes. NHIRD has been extensively used in several published epidemiological research studies. The Longitudinal Health Insurance Database is a data subset comprising 1 million randomly sampled data from NHIRD.

\section{Patients}

Patients with RA were identified from NHIRD using the ICD-9-CM code 714.0. These codes have been adopted in epidemiological studies that used NHIRD data $(9,10)$. We first selected patients who had received the first diagnosis of RA between January 1, 2000, and December 31, 2012. RA is typically treated with two types of anti-inflammatory agents, nonsteroidal anti-inflammatory drugs (NSAIDs) in combination with glucocorticoids and disease-modifying antirheumatic drugs (DMARDs) (11). In Taiwan, several
DMARDs are frequently used for treating patients with RA, including hydroxychloroquine, methotrexate (MTX), and sulfasalazine. The NHI Administration in Taiwan recommends that rheumatologists should prescribe biological DMARDs for patients with RA in whom treatment with conventional DMARDs for at least 6 months is not effective (12). To improve the diagnostic validity of the analysis, this study only included patients with RA under DMARDs for $\geq 30$ days and those aged $\geq 20$ years. The index date was the date of the patient's first RA diagnosis. To define the appropriate cohort of RA patients, patients with comorbidities known to induce alopecia comorbidities such as systemic lupus erythematosus (ICD-9CM code 710.0), cancer (ICD-9-CM codes 140-208), atopic dermatitis (ICD-9-CM code 691), and psoriasis (ICD-9-CM code 696) were excluded $(13,14)$. The enrollees with alopecia areata (AA) were identified by a diagnosis by dermatologists by using the ICD-9-CM code 704.01. We also excluded patients who received a diagnosis of alopecia areata (ICD-9-CM code 704.0) before the index date.

\section{Matching and Covariates}

We conducted frequency matching to select individuals without RA as controls for comparison to increase the statistical power and control for potential confounding factors. Patients with RA and the comparison controls were 1:10 age and sex matched to obtain the same index date in both cohorts (Figure 1). We also estimated whether participants had any of these comorbidities: hypertension (ICD-9-CM codes 401-405), hyperlipidemia (ICD9-CM codes 272.0-272.4), diabetes (ICD-9-CM code 250), and thyroid disorders (ICD-9-CM codes 240-246). The medical records of corticosteroid and DMARD prescription, including methotrexate and hydroxychloroquine, for $\geq 30$ days were also retrieved from ambulatory and patient claims data for patients and controls. After 1:1 propensity score matching for age, sex, hypertension (ICD-9-CM codes 401-405), hyperlipidemia (ICD-9-CM codes 272.0-272.4), diabetes (ICD-9-CM code 250), thyroid disease (ICD-9-CM codes 240-246), mental disorder (ICD-9-CM code 290-319), and corticosteroid use in patients, we conducted the same matching on the comparison cohort to reduce the heterogeneity between the two groups. Information on comorbidity and medication use was determined at baseline and considered to be covariates in the multivariate analysis.

\section{Outcome Measurement}

Outcome variables included AA (ICD-9-CM code 704.0) development during the follow-up period. To improve the diagnostic validity of the analysis, this study included only those patients who received a diagnosis of alopecia by a dermatologist. The patients and controls were followed until the occurrence of alopecia, December 31, 2013, or withdrawal from the national insurance system, whichever occurred first.

\section{Statistical Analysis}

We used the $\chi^{2}$ test and Student's $t$-test to analyze the demographic data between the RA and non-RA groups and the Cox proportional-hazard regression models to calculate hazard ratios (HRs). The Kaplan-Meier curve was used to 


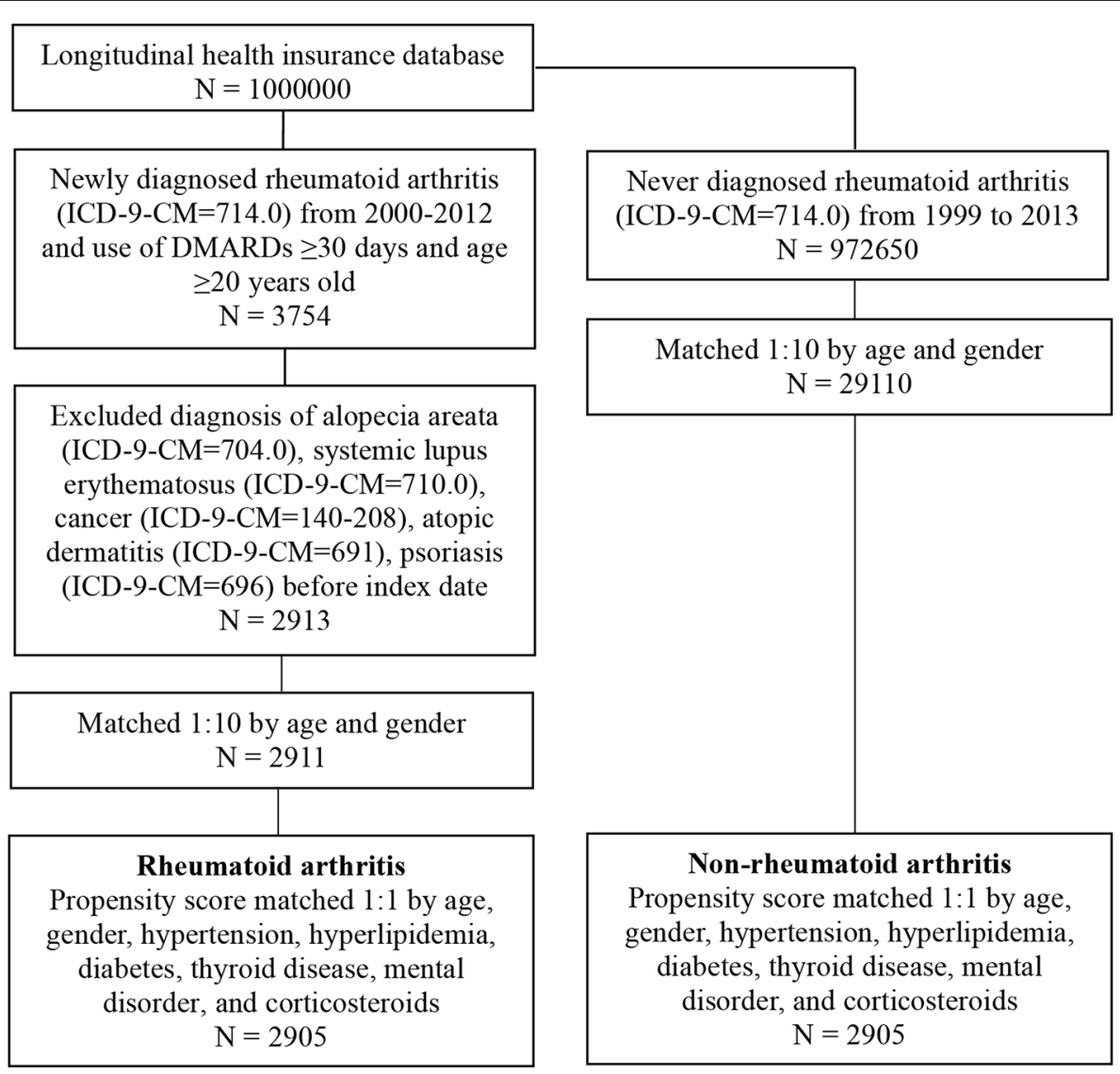

FIGURE 1 | Study flowchart.

calculate the cumulative probability of AA in patients with RA. All data analyses were performed using SPSS (version 18.0; SPSS, Chicago, IL, USA), and $P<0.05$ was considered statistically significant.

\section{RESULTS}

Before propensity score matching, 2,911 patients were included in the RA cohort and 29,100 individuals without RA were included in the control cohort. The RA and control cohorts did not significantly differ in terms of sex and age (Table 1). Patients with RA had a higher prevalence of pre-existing comorbidities such as hypertension, hyperlipidemia, chronic obstructive pulmonary disease, thyroid disease, and mental disorder $(P<0.05)$. Finally, after propensity score matching, 2,905 patients were included in the RA cohort and 2,905 participants with no RA diagnosis were included in the comparison cohort. The age in the propensity score-adjusted cohort was not statistically different between groups.

Table 2 presents the incidence density rate and adjusted HR (aHR) of alopecia in the study population. Patients with RA and controls were followed for 22,276 and 25,792 personyears, respectively. The overall alopecia incidence density rate was significantly higher in patients with RA than in controls (1.7 vs. 0.6 per 1,000 person-years). After adjustments for the covariates, alopecia risk was higher in patients with RA than in controls $(\mathrm{aHR}=2.64,95 \%$ confidence interval [CI] $=1.47-4.76)$. Other risk factors for AA included young age (20-40 years) and thyroid disease. Those aged $\geq 65$ years had a lower AA risk than those aged 20-40 years $(\mathrm{aHR}=$ $0.15,95 \% \mathrm{CI}=0.03-0.67)$. Patients with oral corticosteroids treatment had a lower alopecia risk (aHR $=0.57,95 \% \mathrm{CI}$ $=0.32-1.00)$ than those without. Table 3 presents the sexand age-stratified effect of RA on alopecia. Female patients with RA probably had a higher AA risk than female patients without RA. Overall, patients with RA aged $20-40$ or $\geq 40$ years exhibited increased alopecia risk. Figure 2 presents the KaplanMeier curve, which indicates a higher cumulative incidence of alopecia in patients with RA than in controls $(P=0.001$, log-rank test).

\section{DISCUSSION}

This is the first nationwide population-based study to assess the association between RA and alopecia risk. This study demonstrated important findings. First, we found a 2.6-fold 
TABLE 1 | Demographic characteristics of rheumatoid arthritis and non-rheumatoid arthritis.

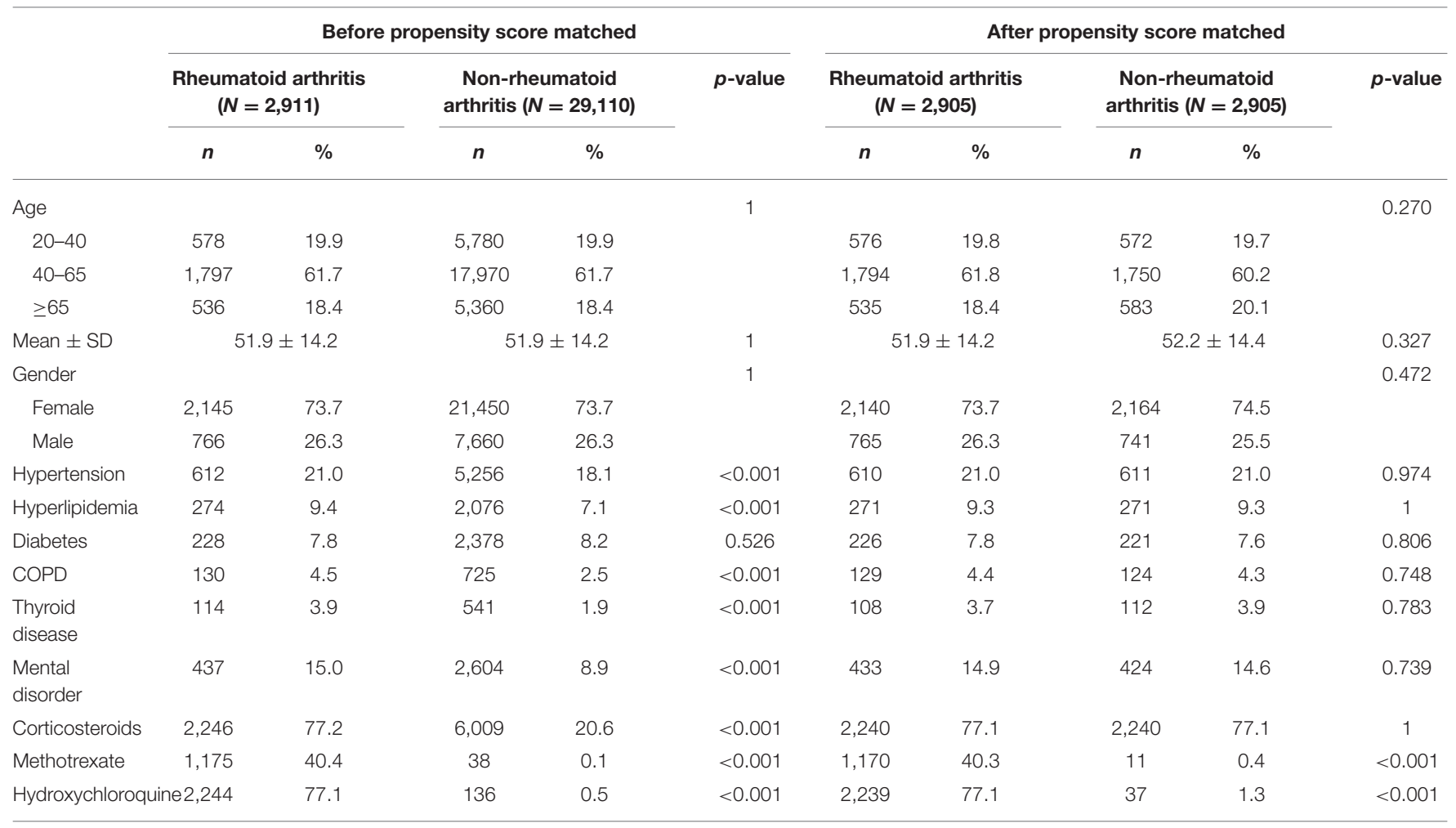

TABLE 2 | Comparison of incidence and HR of alopecia between patients with and without rheumatoid arthritis by Cox proportional hazard model.

\begin{tabular}{|c|c|c|c|c|c|c|c|}
\hline & $\begin{array}{l}\text { No. of alopecia } \\
\text { areata }\end{array}$ & $\begin{array}{c}\text { Observed } \\
\text { person-years }\end{array}$ & $\begin{array}{l}\text { Incidence Density } \\
\text { (Per 1,000 person-years) }\end{array}$ & Crude HR & $95 \% \mathrm{Cl}$ & Adjusted $\mathrm{HR}^{\dagger}$ & $95 \% \mathrm{Cl}$ \\
\hline \multicolumn{8}{|c|}{ Rheumatoid arthritis } \\
\hline Yes & 37 & 22,276 & 1.7 & 2.67 & $1.49-4.81$ & 2.64 & $1.47-4.76$ \\
\hline \multicolumn{8}{|l|}{ Age } \\
\hline$\geq 65$ & 2 & 7,846 & 0.3 & 0.10 & $0.02-0.41$ & 0.15 & $0.03-0.67$ \\
\hline \multicolumn{8}{|l|}{ Gender } \\
\hline Female & 38 & 36,190 & 1.1 & 1 & & 1 & \\
\hline Male & 15 & 11,879 & 1.3 & 1.21 & $0.67-2.21$ & 1.11 & $0.61-2.05$ \\
\hline Hypertension & 4 & 8,921 & 0.4 & 0.35 & $0.13-0.98$ & 0.77 & $0.26-2.28$ \\
\hline Corticosteroids & 34 & 38,626 & 0.9 & 0.44 & $0.25-0.77$ & 0.57 & $0.32-1.00$ \\
\hline
\end{tabular}

${ }^{\dagger}$ Adjusted for age, gender, hypertension, hyperlipidemia, diabetes, thyroid disease, mental disorder, and corticosteroids.

increased alopecia risk in patients with RA compared with the population without RA, especially in younger age groups (2040 years). Second, both RA and thyroid disease occurred significantly more frequently in patients with alopecia. Furthermore, we noted a probably negatively associated determinants of alopecia in oral corticosteroids for patients with RA.

Although patients with RA in this study had a higher prevalence of comorbidities and coexistent conditions associated with alopecia development than the comparison 
TABLE 3 | Subgroup analysis of Cox proportional hazard model stratified by sex and age.

\begin{tabular}{|c|c|c|c|c|c|}
\hline \multicolumn{2}{|c|}{$\begin{array}{l}\text { Rheumatoid } \\
\text { arthritis }\end{array}$} & \multicolumn{2}{|c|}{$\begin{array}{c}\text { Non-rheumatoid } \\
\text { arthritis }\end{array}$} & \multirow[b]{2}{*}{ HR } & \multirow[b]{2}{*}{$95 \% \mathrm{Cl}$} \\
\hline$N$ & $\begin{array}{c}\text { No. of } \\
\text { alopecia } \\
\text { areata }\end{array}$ & $N$ & $\begin{array}{l}\text { No. of } \\
\text { alopecia } \\
\text { areata }\end{array}$ & & \\
\hline
\end{tabular}

\begin{tabular}{|c|c|c|c|c|c|c|}
\hline \multicolumn{7}{|l|}{ Age } \\
\hline $20-40$ & 576 & 18 & 572 & 9 & 2.28 & $1.03-5.08$ \\
\hline \multirow[t]{2}{*}{$\geq 40$} & 2,329 & 19 & 2,333 & 7 & 3.13 & $1.31-7.45$ \\
\hline & & & & & \multicolumn{2}{|c|}{$p$ for interaction $=0.144$} \\
\hline \multicolumn{7}{|l|}{ Gender } \\
\hline Female & 2,140 & 29 & 2,164 & 9 & 3.75 & $1.77-7.92$ \\
\hline Male & 765 & 8 & 741 & 7 & 1.29 & $0.47-3.57$ \\
\hline
\end{tabular}

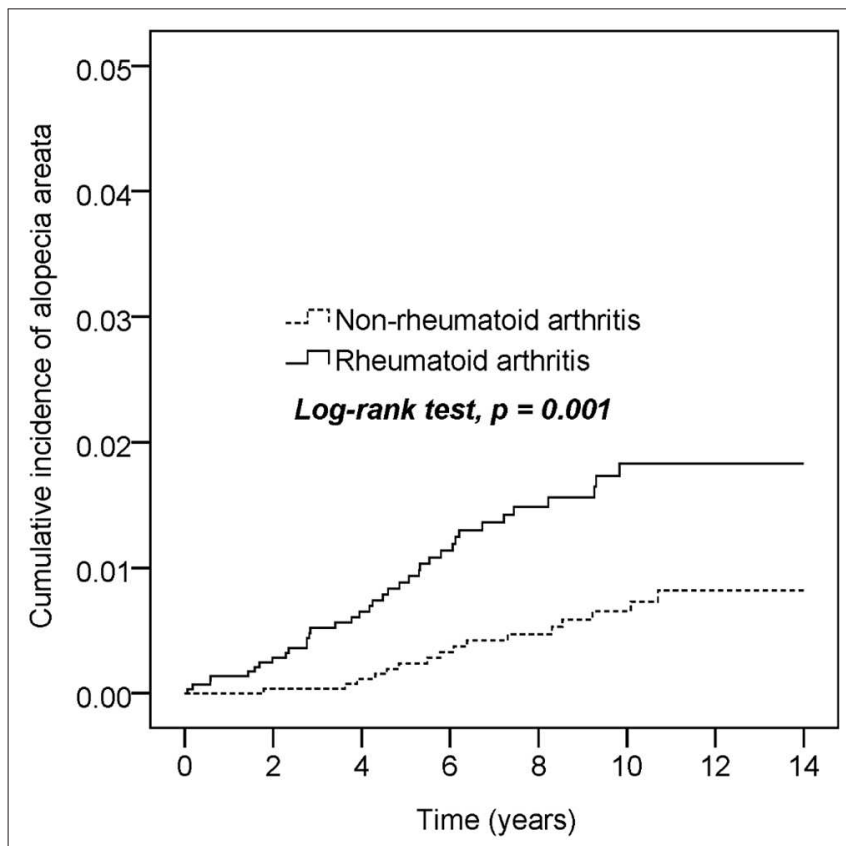

FIGURE 2 | Cumulative incidence comparison of alopecia areata for patients with or without rheumatoid arthritis.

cohort, RA remains an independent risk factor for alopecia after adjustments for covariates. Several biological lines of evidence support this statistical outcome. Petukhova et al. (15) demonstrated that 139 single-nucleotide polymorphisms are associated with alopecia and mostly related to inflammation and immunomodulation. Genome-wide association studies have revealed that autoimmune diseases (e.g., alopecia, RA, and type 1 diabetes mellitus) involve similar pathological changes within the Janus kinase and signal transducer and activator of transcription signaling (16). Mouse model studies have also indicated the role of an identical pathogen involved in this signaling in alopecia $(17,18)$.

Autoimmune diseases, including RA, could be a crucial risk factor for alopecia. Moreover, Cox proportional-hazards regression analysis results in the present study demonstrated a significant increase in alopecia risk in not only patients with RA but also patients with thyroid disease. Moreover, alopecia incidence rate increased in all age groups in patients with RA compared with those without. This finding is consistent with previous studies that showed that thyroid disease is the most common autoimmune comorbidity in patients with alopecia $(19,20)$. A retrospective study on children with alopecia screened for thyroid function revealed that $20 \%$ of the analyzed patients had abnormal thyroid test results (21). Several studies have found the presence of thyroid autoantibodies in 15-20\% of patients with alopecia $(2,22,23)$. Our study clarified that thyroid disease and RA were most significantly associated with alopecia. Previous studies have concluded that thyroid function screening should be considered, particularly in alopecia patients with a history of Down syndrome or atopy or a family history of thyroid disease (2). Furthermore, our study results can help clinicians identify and understand comorbidities, which can enhance their ability to recommend screening and preventative measures.

In the general population, alopecia can develop at any age; however, in $82.6-88 \%$ of the cases, it develops by the age of 20 years $(24,25)$. This study demonstrated that the young age was associated with a lower alopecia risk. Genetic (inherited) factors may play a role in the interaction between alopecia and age (26). Early onset of AA seems to indicate the underlying genetic background. Chu et al. (13) recently found that AA onset age predicts the associated autoimmune diseases. Almost $40 \%$ of individuals aged $<30$ years with AA have at least one family member who has been diagnosed with the same disorder (27).

A notable finding of the current study is that oral glucocorticoid use was probably associated with a reduced AA risk. Systemic glucocorticoids are occasionally prescribed as a temporary measure to slow hair loss in patients with rapidly progressing extensive hair loss $(28,29)$. By contrast, some DMARDs used in RA management may present adverse effects such as hair loss $(30,31)$. Alopecia was reported as an adverse effect of treatment with methotrexate. In the present study, the clinical suitability of glucocorticoids or DMARDs for disease activity assessment remains uncertain.

We found significant associations between RA and alopecia, indicating the crucial role of RA in alopecia. Although our study also demonstrated other determinants of alopecia included thyroid disease, young age (positively associated), and glucocorticoid therapy (negatively associated), there are some potential limitations: First, because of the large variation in clinical manifestations and diagnostic definitions, RA diagnosis may be disregarded by clinicians. However, the accuracy of RA and AA diagnoses in NHIRD has been validated previously $(9,10,13)$. Second, NHIRD does not include data on covariates, such as family history, personal lifestyle, social adversity, laboratory data, environmental factors, and disease activity. The RA disease activity may have an impact in alopecia. The lack of data on disease activity and glucocorticoid/DMARD doses is also a limitation.

In conclusion, RA is associated with a higher incidental alopecia risk. Alopecia risk must be carefully evaluated in patients 
with RA. Clinically, understanding these findings is of high relevance for damage prevention. Further prospective studies should investigate the cost-effectiveness of screening for RA in patients with alopecia.

\section{DATA AVAILABILITY STATEMENT}

The datasets generated for this study are available on request to the corresponding author.

\section{ETHICS STATEMENT}

This study was approved by the Chung Shan Medical University Hospital Ethics Committee.

\section{REFERENCES}

1. Wasserman D, Guzman-Sanchez DA, Scott K, McMichael A. Alopecia areata. Int J Dermatol. (2007) 46:121-31. doi: 10.1111/j.1365-4632.2007. 03193.x

2. Patel D, Li P, Bauer AJ, Castelo-Soccio L. Screening guidelines for thyroid function in children with alopecia areata. JAMA Dermatol. (2017) 153:130710. doi: 10.1001/jamadermatol.2017.3694

3. Huang KP, Mullangi S, Guo Y, Qureshi AA. Autoimmune, atopic, and mental health comorbid conditions associated with alopecia areata in the United States. JAMA Dermatol. (2013) 149:789-94. doi: 10.1001/jamadermatol.2013.3049

4. Conic RZ, Miller R, Piliang M, Bergfeld W, Mesinkovska NA. Comorbidities in patients with alopecia areata. J Am Acad Dermatol. (2017) 76:755-7. doi: 10.1016/j.jaad.2016.12.007

5. Lee NR, Kim B-K, Yoon NY, Lee S-Y, Ahn S-Y, Lee W-S. Differences in comorbidity profiles between early-onset and late-onset alopecia areata patients: a retrospective study of 871 Korean patients. Ann Dermatol. (2014) 26:722-6. doi: 10.5021/ad.2014.26.6.722

6. Lim CP, Severin RK, Petukhova L. Big data reveal insights into alopecia areata comorbidities. J Invest Dermatol Symp Proc. (2018) 19:S57-S61. doi: 10.1016/j.jisp.2017.10.006

7. Rudan I, Sidhu S, Papana A, Meng S-J, Xin-Wei Y, Wang W, et al. Global Health Epidemiology Reference Group (GHERG). Prevalence of rheumatoid arthritis in low- and middle-income countries: a systematic review and analysis. J Glob Health. (2015) 5:010409. doi: 10.7189/jogh.05. 010409

8. McInnes IB, Schett G. The pathogenesis of rheumatoid arthritis. N Engl J Med. (2011) 365:2205-19. doi: 10.1056/NEJMra1004965

9. Chung WS, Peng CL, Lin CL, Chang YJ, Chen YF, Chiang JY, et al. Rheumatoid arthritis increases the risk of deep vein thrombosis and pulmonary thromboembolism: a nationwide cohort study. Ann Rheum Dis. (2014) 73:1774-80. doi: 10.1136/annrheumdis-2013203380

10. Tam HW, Chen CM, Leong PY, Chen CH, Li YC, Wang YH, et al. Methotrexate might reduce ischemic stroke in patients with rheumatoid arthritis: a population-based retrospective cohort study. Int J Rheum Dis. (2018) 21:1591-9. doi: 10.1111/1756-185X.13267

11. Yazici Y. Treatment of rheumatoid arthritis: we are getting there. Lancet. (2009) 374:178-80. doi: 10.1016/S0140-6736(09)60792-3

12. Chou MH, Wang JY, Lin CL, Chung WS. DMARD use is associated with a higher risk of dementia in patients with rheumatoid arthritis: a propensity score-matched case-control study. Toxicol Appl Pharmacol. (2017) 334:21722. doi: 10.1016/j.taap.2017.09.014

13. Chu SY, Chen YJ, Tseng WC, Lin MW, Chen TJ, Hwang CY, et al. Comorbidity profiles among patients with alopecia areata: the importance of onset age, a nationwide population-based study. J Am Acad Dermatol. (2011) 65:949-56. doi: 10.1016/j.jaad.2010.08.032

\section{AUTHOR CONTRIBUTIONS}

JW designed the research. Y-JC and Y-HL were responsible for drafting the manuscript and collected the data. Y-HW and $\mathrm{P}-\mathrm{YL}$ analyzed the data. Y-JC and JW reviewed and revised the manuscript. Y-HL, P-YL, and Y-HW reviewed the manuscript. All authors approved this version to be published.

\section{FUNDING}

The funders had no role in study design, data collection and analysis, decision to publish, or preparation of the manuscript. This study is supported by the Chung Shan Medical University Hospital grant number CSH-2020-C-007.

14. Chen CC, Chang YT, Liu HN, Chen YJ. Cancer risk in patients with alopecia areata: a nationwide population-based matched cohort study. Cancer Med. (2018) 7:2153-9. doi: 10.1002/cam4.1448

15. Petukhova L, Duvic M, Hordinsky M, Norris D, Price V, Shimomura Y, et al. Genomewide association study in alopecia areata implicates both innate and adaptive immunity. Nature. (2010) 466:113-7. doi: 10.1038/nature 09114

16. Betz RC, Petukhova L, Ripke S, Huang H, Menelaou A, Redler S, et al. Genome-wide meta-analysis in alopecia areata resolves HLA associations and reveals two new susceptibility loci. Nat Commun. (2015) 6:5966. doi: $10.1038 /$ ncomms 6966

17. Divito SJ, Kupper TS. Inhibiting Janus kinases to treat alopecia areata. Nat Med. (2014) 20:989-90. doi: 10.1038/nm.3685

18. Xing L, Dai Z, Jabbari A, Cerise JE, Higgins CA, Gong W, et al. Alopecia areata is driven by cytotoxic T lymphocytes and is reversed by JAK inhibition. Nat Med. (2014) 20:1043-9. doi: 10.1038/nm.3645

19. Kahaly GJ, Frommer L. Polyglandular autoimmune syndromes. J Endocrinol Invest. (2018) 41:91-8. doi: 10.1007/s40618-017-0740-9

20. Thomas EA, Kadyan RS. Alopecia areata and autoimmunity: a clinical study. Indian J Dermatol. (2008) 53:70-4. doi: 10.4103/0019-5154. 41650

21. Bertolini M, Zilio F, Rossi A, Kleditzsch P, Emelianov VE, Gilhar A, et al. Abnormal interactions between perifollicular mast cells and CD8+ T-cells may contribute to the pathogenesis of alopecia areata. PLoS One. (2014) 9:e94260. doi: 10.1371/journal.pone.0094260

22. Saylam Kurtipek G, Cihan FG, Erayman Demirbaş S, Ataseven A. The Frequency of autoimmune thyroid disease in alopecia areata and vitiligo patients. Biomed Res Int. (2015) 2015:435947. doi: 10.1155/2015/ 435947

23. Bertolini M, Rossi A, Paus R. Cover image: are melanocyte associated peptides the elusive autoantigens in alopecia areata? Br J Dermatol. (2017) 176:1106. doi: $10.1111 /$ bjd. 15288

24. Yang S, Yang J, Liu JB, Wang HY, Yang Q, Gao M, et al. The genetic epidemiology of alopecia areata in China. Br J Dermatol. (2004) 151:16-23. doi: 10.1111/j.1365-2133.2004.05915.x

25. Tan E, Tay YK, Goh CL, Giam YC. The pattern and profile of alopecia areata in Singapore - a study of 219 Asians. Int J Dermatol. (2002) 41:748-53. doi: 10.1046/j.1365-4362.2002.01357.x

26. Ahmed A, Almohanna H, Griggs J, Tosti A. Genetic hair disorders: a review. Dermatol Ther. (2019) 9:421-48. doi: 10.1007/s13555-019-0313-2

27. Harvard Health Publishing. Alopecia Areata. (2019). Available online at: https://www.health.harvard.edu/a_to_z/alopecia-areata-a-to-z (accessed April 17, 2020).

28. Vano-Galvan S, Hermosa-Gelbard A, Sanchez-Neila N, Miguel-Gómez L, Saceda-Corralo D, Rodrigues-Barata R, et al. Pulse corticosteroid therapy with oral dexamethasone for the treatment of adult alopecia totalis and universalis. J Am Acad Dermatol. (2016) 74:1005-7. doi: 10.1016/j.jaad.2015. 12.026 
29. Cowley BJ, Dong J. Use of oral corticosteroids in the treatment of alopecia areata. Arch Dis Child. (2020) 105:96-8. doi: 10.1136/archdischild-2019-317956

30. Gottenberg JE, Venancie PY, Mariette X. Alopecia areata in a patient with rheumatoid arthritis treated with leflunomide. J Rheumatol. (2002) 29:1806-7.

31. Kanameishi S, Dainichi T, Endo Y, Otsuka A, Tanioka M, Kabashima K. Successful hair regrowth in an acute diffuse form of alopecia areata during oral tacrolimus treatment in a patient with rheumatoid arthritis. J Eur Acad Dermatol Venereol. (2017) 31:e137-8. doi: 10.1111/jdv. 13879
Conflict of Interest: The authors declare that the research was conducted in the absence of any commercial or financial relationships that could be construed as a potential conflict of interest.

Copyright (c) 2020 Chang, Lee, Leong, Wang and Wei. This is an open-access article distributed under the terms of the Creative Commons Attribution License (CC BY). The use, distribution or reproduction in other forums is permitted, provided the original author(s) and the copyright owner(s) are credited and that the original publication in this journal is cited, in accordance with accepted academic practice. No use, distribution or reproduction is permitted which does not comply with these terms. 